Conclusions Longer hospital stay prior to PICU admission, steroid use, mechanical ventilation and CVC were associated with becoming MRSA affected.

\section{USUAL MICROBE STRAINS IN A PEDIATRIC INTENSIVE CARE UNIT}

doi:10.1136/archdischild-2012-302724.1572

E Blevrakis, T Tavladaki, AM Spanaki, E Vasilaki, MD Fitrolaki, E Geromarkaki, S llia, G Briassoulis. PICU, University Hospital of Heraklion, Heraklion, Greece

Background and Aim Healthcare-associated infections (HAIs) are an important cause of morbidity and mortality among critically ill children. This retrospective surveillance study was performed to estimate the burden of HAIs in a paediatric intensive care unit of our country. During the $61 / 2$ years study 660 patients were treated in the PICU for $>48$ hours.

Methods A retrospective study of healthcare associated pneumonia (HAP) blood stream and urinary tract infections is recorded.

Results The crude infection rate were healthcare-associated pneumonia, bloodstream and urinary tract infections and approximately above the two thirds came from microbes isolated from bronchial aspirations. Table 1 positive cultures.

Pseudomonas Aeruginosa was the commonest infected bacterium followed by Staphylococcus Aureus and Klebsiella Pneumoniae in very low percentage. Table 2 microbes strain.

\section{Abstract 1572 Table 1 Positive cultures}

\begin{tabular}{lcc}
\hline BAL & $\mathbf{6 8 . 7 5 \%}$ & $\mathbf{8 1 \%}$ \\
\hline BLOODSTREAM & $22,2 \%$ & $81 \%$ \\
URINE & $9,76 \%$ & $81 \%$ \\
OTHER SYSTEM & & $19 \%$ \\
\hline
\end{tabular}

Abstract 1572 Table 2 Microbes strains

\begin{tabular}{ll}
\hline Pseudomonas Aeruginosa & $\mathbf{3 9 \%}$ \\
\hline Staphylococcus Aureus & $5 \%$ \\
Klebsiella Pneumoniae & $4,65 \%$ \\
\hline
\end{tabular}

Conclusions The above data are similar in those in literature and active surveillance is essential to reduce the burden of HAIs and intensive efforts have already began.

\section{SEPSIS AND MULTIPLE ORGAN DYSFUNCTIONS IN OUR PICU}

doi:10.1136/archdischild-2012-302724.1573

E Celaj, I Bakalli, E Kola, R Lluka, A Vula, D Sala, S Sallabanda. PICU, UHC 'Mother Teresa', Tirana, Albania

Background and Aims Multiple organ dysfunction (MODS) may occur during septic disease and it associated with high mortality. The final outcome depends of many factor such as the age, the host response to infection, the site and type of infection, the development of shock, the underlying disease, and the number of failed organs.

The Aim of the study was to determine the frequency of sepsis and MODS in our PICU, define the clinical and laboratory features of affective children and evaluate their outcomes.

Methods A retrospective analysis of children presenting with sepsis and MODS in our PICU between January - December 2011.

Results During the period of study, 478 patients were admitted in our PICU. Sepsis and MODS occurred in $10.9 \%(n=52)$ of hospital admissions. Girls were $63 \%$ and boys $37 \%$. The overall mortality was $36.5 \%$. Three children died during the first $24 \mathrm{~h}$ after the admission and for the rest the mean hospital stay was 8.4 days.

The most frequent organs involved were respiratory, cardiovascular, hematological and neurological. The organs that were less frequently involved were the gastrointestinal, renal and hepatic organs.

The survival depended on the number of affected systems. When two systems were involved the survival was $84 \%$, three systems $34 \%$, four systems $25 \%$ and five or more with no survival.

Conclusions Sepsis and MODS were associated with increased severity of illness.

The mortality rate associated with multiple organ system failure in pediatric patients is high. Mortality increased with increasing number of organ dysfunctions.

\section{HEPATOADRENAL SYNDROME IN EGYPTIAN CHILDREN WITH LIVER CIRRHOSIS WITH AND WITHOUT SEPSIS}

doi:10.1136/archdischild-2012-302724.1574

A Elfaramawy. Pediatrics, Ain Shams University, Cairo, Egypt

The similarities between septic shock and liver failure led to the proposal of the term hepatoadrenal syndrome. This study aimed to evaluate children with liver cirrhosis for the presence of adrenal insufficiency especially during critical illness. It was designed to evaluate adrenal function for 24 children with liver cirrhosis of various etiologies by measuring basal cortisol level and measuring the peak level after 30 minutes of short low dose ACTH stimulation test. They were categorized to; group 1 included 12 patients with sepsis and group 2 included 12 patients without sepsis. It was found in this study that no one of group1or group $2 \mathrm{had}$ absolute adrenal insufficiency; however 11 patients, $8(66.6 \%)$ of group 1 and 3 patients (25\%)of group 2 had relative adrenal insufficiency (RAI) as the increment detected in cortisol level after stimulation was $<9 \mu \mathrm{gm} / \mathrm{dL}$. Most of patients with RAI $(72.7 \%)$ were categorized as having Child -Pugh $\mathrm{C}$ liver cirrhosis. The presence of ascites, high temperature, high $\mathrm{C}$ reactive protein, neutrophilia, high ALT, AST, high total bilirubin, prolonged INR and lower albumin were all risk factors associated with the occurrence of RAI. Survival rate in patients with normal adrenal function $(92 \%)$ was significantly better than in patients with RAI $(27 \%)(p=0.02)$. It was concluded from this study that a high incidence of relative adrenal insufficiency was found in children with liver cirrhosis. It was more common in the presence of sepsis, related to the degree of liver cirrhosis and carried a bad prognosis.

\section{LUMBAR PUNCTURE(LP) IN INFANTS AND CHILDREN WITH SUSPECTED MENINGITIS:REGIONAL TRENDS OVER 15 YEARS}

doi:10.1136/archdischild-2012-302724.1575

Z Barsoum, E Kieran, J Powell, N Barrett, NO Connell, RK Philip-Limerick. Paediatrics, Mercy University Hospital, Cork, Ireland

Background and Aims Lumbar puncture (LP) has been long considered a useful tool to assist in the diagnosis of meningitis. We aim to study the trends of successful LPs in an Irish Regional hospital and to further analyse, timing of LP, microbiological and serological positive yield and the potential impact on treatment.

Methods A retrospective analysis of laboratory data of all successful LPs for suspected meningitis among the $0-15$ year age group was performed from July 1996 to December 2010 at University Hospital-Limerick. Repeat studies and samples from the Regional Maternity hospital were separately analysed.CSF studies for other conditions were excluded.HIPE data on meningitis admissions and supportive laboratory data were collated. Hospital Audit commitee approval was obtained. 
Results 1487 successful LPs were performed from 1996 to 2010.Of these 646 procedures were performed from 1996 to 2000 and 463 samples obtained from 2001 to 2005 .Only 378 LPs were performed from 2006 to 2010. Of those with CSF analysed,967(65\%) were 2 years or younger. $53 \%$ of LPs were performed at night time. Only 18 patients had bacterial meningitis confirmed on $\operatorname{CSF}(1.2 \%)$ from 2001 to 2010 and $60 \%$ were infants.LP was performed before the use of antibiotics in $15 \%$ of cases. Nisseria meningitidis B and Stryptococcus pneumoniae were the leading causes of meningitis (38\%\&27\% respectively).Among those with confirmed meningitis on CSF, serum PCR was positive in $77 \%$ while CSF PCR was positive in $100 \%$ of cases.

Conclusion Incidence of bacterial meningitis is decreasing mainly due to effective vaccination programmes.LP, even in the post vaccination era with early use of emperical antibiotics, still has a role in the diagnosis of meningitis.

\section{EFFECT OF DEXAMETHASONE THERAPY ON THE EXPRESSION OF MACROPHAGE MIGRATION INHIBITORY FACTOR IN CRITICALLY ILL CHILDREN WITH SEPTIC SHOCK}

doi:10.1136/archdischild-2012-302724.1576

MGE Mansour, MAM Tarek, Y Zayton, A Zein. Ain Shams University, Cairo, Egypt

Background and objectives Macrophage migration inhibitory factor (MIF) is a proinflammatory cytokine that plays a major role in the pathogenesis of sepsis. The goal of this study was to determine the effect of exogenous glucocorticoid treatment on MIF expression.

Design and setting: Prospective, randomized, double-blinded, interventional single-center study.

Methods Thirty patients with septic shock were prospectively randomized to receive 3 doses of $0.2 \mathrm{mg} / \mathrm{kg}$ of dexamethasone (Intervention group) or equal doses of saline (Placebo group). Sequential Organ Failure Assessment (SOFA) and Pediatric Logistic Organ Dysfunction (PELOD) scores were recorded daily in both groups. Seven and 28-day Mortality were recorded as early and late endpoints of the study. Baseline and follow-up levels of MIF were measured by ELISA for all enrolled subjects.

Results Mean baseline MIF values were $86 \pm 17,82 \pm 21,10.8 \pm 5 \mathrm{ng} /$ $\mathrm{mL}$ for Intervention, Placebo and control subjects respectively; $p<0.001$. Follow up MIF showed significant reduction in both patients groups compared to baseline levels, with more significant reduction in Intervention group (23\% reduction) compared to Placebo group (13\% reduction); $\mathrm{p}<0.01$. SOFA score showed significant worsening in Placebo group; compared to Intervention group. PELOD score showed significant increase in Intervention group compared to highly significant worsening in Placebo group. Sevenday mortality was significantly higher in Placebo group (53.3\%) compared to Intervention group (20\%); while 28-day mortality showed insignificant change.

Conclusions Early treatment with dexamethasone lead to more significant reduction in MIF levels than placebo, with less worsening of organ dysfunction and improved seven-day mortality with no effect on 28-day mortality.

\section{HDL AND LDL BLO0D LEVELS ARE OPPOSED TO TRIGLYCERIDES AND GLUCOSE IN SEVERE SEPSIS}

doi:10.1136/archdischild-2012-302724.1577

${ }^{1} \mathrm{MD}$ Fitrolaki, ${ }^{2} \mathrm{H}$ Dimitriou, ${ }^{1} \mathrm{~S}$ llia, ${ }^{1} \mathrm{AM}$ Spanaki, 'E Geromarkaki, ${ }^{1}$ E Vasilaki, ${ }^{1} \mathrm{E}$ Tavladaki, 'E Blevrakis, 'G Briassoulis. 'Paediatric Intensive Care Unit, University Hospital of Crete, ${ }^{2}$ Paediatric Haematology Oncology, University of Crete, Heraklion, Greece

Introduction Lipoproteins were shown to neutralize LPS and to exert direct anti-inflammatory actions. Neutrophil CD64-expression (nCD64) was shown to be an early inflammatory sign.
Objectives To examine whether plasma concentrations of high (HDL)- or low (LDL)-density lipoproteins are related to nCD64, triglycerides, glucose, severity of illness (PRISM, PELOD), length of stay (LOS) or mechanical ventilation (LOMV), and mortality in children with sepsis (S) and severe sepsis/septic shock (SS) compared to those with trauma ( $\mathrm{T}$ ) or healthy controls (C).

Methods 48 children were classified into 4 groups of SS, S, T, and C (12 each). Blood samples were collected on 3 consecutive days following admission.

Results On day $1 \mathrm{HDL}, \mathrm{LDL}$, and cholesterol were found to be significantly lower in SS and S compared to C (29.5 \pm 2.7 and $27.3 \pm 4.2$, vs. $58.8 \pm 5.8 \mathrm{mg} / \mathrm{dl}, \mathrm{p}<0.001,33.4 \pm 5.8$ and $66.2 \pm 9$, vs. $98.1 \pm 6.5$ $\mathrm{mg} / \mathrm{dl}, \mathrm{p}<0.001,100.6 \pm 9$ and $125.2 \pm 16$, vs. $171.3 \pm 7 \mathrm{mg} / \mathrm{dl}, \mathrm{p}<0.001$ respectively). Opposite trends followed triglycerides (209 \pm 61 and $151.7 \pm 25$, vs. $71.8 \pm 10 \mathrm{mg} / \mathrm{dl}, \mathrm{p}=0.007)$ and glucose $(117 \pm 8$ and $115 \pm 12$, vs. $83 \pm 3 \mathrm{mg} / \mathrm{dl}, \mathrm{p}=0.002)$. HDL and triglycerides differed between S or SS and T ( $<<0.04)$. Cholesterol, HDL, and LDL were negatively related with $\mathrm{nCD} 64$, procalcitonin, CRP, glucose, LOS, and LOMV ( $p<0.05)$, but not with severity of illness. Glucose was positively related to the LOS and PELOD $(p<0.05)$ and triglycerides with CRP $(p<0.05)$

Conclusions Lipoproteins and cholesterol are markedly reduced in severe sepsis, but not in trauma, and are inversely related to nCD64expression, acute phase proteins, glucose, LOS, and LOMV.

\section{THE CORRELATION BETWEEN C-REACTIVE PROTEIN AND SERIOUS BACTERIAL INFECTION IN A TERTIARY PAEDIATRIC EMERGENCY DEPARTMENT: AN OBSERVATIONAL CASE-CONTROLLED COHORT STUDY}

doi:10.1136/archdischild-2012-302724.1578

1,2,3 MJ Barrett, ' $\mathrm{J}$ Pollock, ${ }^{1} \mathrm{~T}$ Harney, ${ }^{1} \mathrm{~S} J$ Yeung, ${ }^{1} \mathrm{~K}$ Laffan, ${ }^{1} \mathrm{E}$ Clarke, ${ }^{1} \mathrm{~S}$ Darcy, ${ }^{4} \mathrm{~T}$ Grant, 'L Melody, 'P Fitzpatrick, 'S Cashman, 'C Howard, 'Z lqbal, ' $\mathrm{F}$ Taib, 'S McCoy, 'S Walsh, ${ }^{1,2,3} \mathrm{R}$ 0'Sullivan. 'Paediatric Emergency Research Unit, Department of Emergency Medicine, Our Lady's Children's Hospital Crumlin (OLCHC); 'National Children's Research Centre, OLCHC; ${ }^{3}$ Department of Paediatrics; ${ }^{4}$ Public Health and Population Science, University College Dublin, Dublin, Ireland

Background and Aims C-Reactive Protein (CRP) is a common component of the blood panel in children being investigated for the considered diagnosis of serious bacterial infection (SBI). We aimed to correlate CRP values to SBI in a paediatric population.

Methods This is a retrospective study from 2007-2009 of all patients with CRP greater than $100 \mathrm{mg} / \mathrm{L}$. Controls were randomly selected age-matched patients with a CRP less than $100 \mathrm{mg} / \mathrm{L}$. SBI was defined as bacterial meningitis, bacteraemia, urinary tract infection, pneumonia, osteomyelitis, septic arthritis, appendicitis or abscess formation confirmed by microbiological investigations and/ or supporting radiology and ultimate clinical diagnosis.

Results 570/10,191 patients had a CRP > 100mg/L. 496 patients were controls 424 patients had SBI. A significant difference between the non-SBI group $(n=642), 35 \pm 68 \mathrm{mg} / \mathrm{L}$ (median \pm standard deviation), compared to the SBI group $(\mathrm{n}=424), 141 \pm 87 \mathrm{mg} / \mathrm{L}$, existed. The absolute and ratio risk of SBI increased consistently with rising CRP values (Table 1).

Abstract 1578 Table 1 Incidence, Absolute \& Ratio Risks for SBI

\begin{tabular}{lcccc}
\hline CRP Range & Incidence of SBI & Total cases & Absolute Risk & Ratio Risk \\
\hline $0-20$ & 20 & 248 & $8 \%$ & 0.08 \\
$21-100$ & 39 & 181 & $21 \%$ & 0.27 \\
$101-150$ & 174 & 365 & $48 \%$ & 0.92 \\
$151-200$ & 83 & 134 & $62 \%$ & 1.63 \\
$201-250$ & 43 & 64 & $67 \%$ & 2.03 \\
$251-300$ & 44 & 52 & $84 \%$ & 5.25 \\
$301+$ & 21 & 22 & $95 \%$ & 21.2 \\
\hline
\end{tabular}

